$\mathbb{T}$ periodica polytechnica

Chemical Engineering

$5 6 / 1 ( 2 0 1 2 ) 3 7 \longdiv { 4 8 }$

doi: $10.3311 /$ pp.ch.2012-1.05

web: http://www.pp.bme.hu/ch

(c) Periodica Polytechnica 2012

RESEARCH ARTICLE

\section{Use of colloid chemistry principles to improve efficiency in biodiesel production}

\author{
András Kovács / Chris Ball
}

Received 2012-03-22, accepted 2012-06-20

\begin{abstract}
The principles of colloid chemistry have been employed to improve the efficiency of biodiesel production. Potential sources of material loss caused by colloid chemistry were linked to specific steps in the sequence of unit operations, and literature reviewed for possible solutions. Liquid-liquid equilibrium and trans-esterification experiments with high oleic sunflower and rapeseed oils were conducted using apolar and polar solvents. The kinetics of trans-esterification were improved by both through the modification of interfacial resistance but the shift to final chemical equilibrium was only possible with apolar solvents. The use of an apolar solvent is beneficial in synthesis and product refining alike, though the removal of interfacial resistance between reaction partners, by shifting the reversible transesterification to close to complete conversion in a single step, and by reducing losses in material and profit. The results can be used to design and operate leaner, more efficient biodiesel production systems.
\end{abstract}

\section{Keywords}

biodiesel · colloid chemistry · process optimization · technology retrofit

\section{Acknowledgement}

This work has been supported by TECH09 support of the Hungarian National Technology and Research Authority (NKTH).

\section{András Kovács}

QS Biodiesel Ltd, 1083, Budapest, Szigetvári u. 1, Hungary

e-mail: andras@kukk.hu

\section{Chris Bal}

QS Biodiesel Ltd, 1083, Budapest, Szigetvári u. 1, Hungary

\section{Introduction}

Many biodiesel experts remain broadly optimistic about the future of the biodiesel industry. However, as shown by the recent spate of plant closures and idling of both small and large scale production facilities, the industry has problems, with many operators struggling to be profitable.

The costs of biodiesel production are impacted by far more than simply the costs related to the conversion of tri-glycerides (TG) and free fatty acids (FFA) into fatty acid methyl ester (FAME) fuel. Traditionally cost-saving has focused on identifying lower cost alternative feedstocks, since as the most expensive component, feedstock prices drive profitability [1]. However inexpensive feedstocks typically contain more than $10 \%$ FFA, which is beyond the operational limit of many conventional biodiesel plants. Current production technology usually requires low FFA levels, preferably below $0.5 \%$ or $0.1 \%$ [2] to avoid loss of catalyst due to saponification. As a consequence, many plants are unable to use these alternative feedstocks, leading to the shutdown of large numbers of plants across Europe and the US over the last 2 years.

This has led to an interest in finding approaches that can use feedstocks with high levels of FFA. These have included, beside investing into acid esterification pretreatment, efforts to using heterogeneous catalysts [3] or full catalytic hydrotreatment to convert such feedstocks into clean-burning alkanes. The renewed interest in hydrotreatment resulted in a rebut of Canmet's patents from the 1990s [4]. Severe hydrotreatment of inferior quality secondary feedstocks produces a mixture of alkane hydrocarbons, known as hydrodiesel. This is typically excellent quality fuel extender but can only be produced at higher cost and lower diesel yield by petroleum refineries. Petroleum refineries opted for co-processing petroleum and renewable diesel feedstocks in severe hydrotreatment units.

The aim of the present article is to analyze significant sources of loss in FAME processing, that are mainly related to colloid chemical phenomena. We use the term 'colloid chemistry' to describe the science of the interactions and behavior of a system containing components possibly separated in distinct liquid phases. By focusing on the underlying chemistry, we iden- 
tify potential sources for financial losses which are caused directly by colloid chemistry, and suggest technological solutions for lowering costs and increasing productivity. Specifically we review the effects of colloid chemistry: an essential element of biodiesel production costs, which to date has largely been ignored in biodiesel synthesis and processing.

\section{The role of colloid chemistry in biodiesel production}

In a typical biodiesel production cycle oilseeds and less expensive oleaginous materials are converted into standard quality renewable ester fuel (Fig. 1) The obvious goal of biodiesel processing is to produce it with the lowest possible loss under profitable economic circumstances. Upon revisiting the main sources of loss we concluded that colloid phenomena play decisive role in controlling the final yield of this renewable fuel.

Source A: Losses associated to pressing the oil bearing seeds are related to the bio-colloid nature of seeds. The oil is being encapsulated into a protein network within which the amounts of water and ions vary and by such different degradation conditions pertain. Not only the species, but the conditions of agricultural practice have influence on oil content and quality of the oil. Storage and handling conditions affect further both the properties and the value of the seed. Strict control of humidity, dust and temperature is needed to maintain high levels of oil and prevention of degradation of TG. The main products of such degradation are DG, MG, G and FFA. Efficient pressing depends on proper handling and treating the seed's bio-colloid structure, through dehulling, size reduction, pre-crushing and conditioning to respond to these factors. These operations modify the structure of the seed's protein network to allow more easy squeeze of the oil. If not done, and the pressed cake is not submitted to solvent extraction the amount of residual oil in the pressed cake can be up to $10-20 \%$. Generally, the smaller the capacity of the press the larger the loss.

Source B: Lack of extraction. The bio-colloid nature of the seed sets limit to mechanical squeeze of the encapsulated oil. Unfortunately the majority of small scale operators in Europe lack appropriate solvent extraction facilities. Even though the pre-treatment operations listed under the previous category of loss have also decisive influence on the extent of oil that can be extracted by suitable solvents (hexane, carbon dioxide, etc.). With proper pre-treatment the residual oil content can be reduced to below $1 \%$. The reported most striking losses are generated in energy costs for solvent recycle. These losses are also directly related to the goodness of oil seed pretreatment and the choice of proper solvent extraction technology. Those techniques that use high rate of extraction solvent are more prone to losses in recycling operations than those that employ smaller rates and more efficient contact techniques. Economy to scale limits the use of extraction operations in small and medium capacity plants. Innovative techniques are needed to reduce the level of scale to economy.

Source C: Losses in refining crude, intermediary and inex- pensive secondary feedstocks result by the imperfect separation of the refined and byproduct streams. Inadequate selectivity and solubility of solvents and reagents, adsorption and entrapment of target products in dispersed byproduct stream are the main causes and mechanisms for loss. Losses can reach the level of $20 \%$ in refining vegetable oils for food grade products [5]. Refining losses are typically 5 times the free fatty acid (FFA) content in the crude vegetable oil, mainly because of formation of emulsions in the neutralization and washing steps [6]. As a rule of thumb recommendation of Dorsa and Eiickhoff [7] Miscella refining with $n$-hexane solvent has been reported to reduce refining losses [8] with no record for application in practice. Complex dispersions of the expensive oil and grease stocks with the refining solvents must be managed so as to separate the extracts and other dispersed contaminants efficiently in reduced number of treatment steps.

Sources D and E: Losses in esterification and transesterification. The most imminent colloid chemistry aspect is associated to esterification and trans-esterification of reaction partners from and into mutually partially soluble phases. Starting components, the fatty feedstock and the mixture of reagent and catalysts are not mutually soluble. To conclude in chemical reaction intimate contact of these is typically achieved by creating a dispersion (emulsion) of the reaction partners. Beside the basic chemistry of FAME processing $\{r-1 . .3\}$, that involves a sequence of reversible reactions there are a series of operations typically influenced by colloid phenomena. Intermediary products of di- and mono-glycerides (DG and MG) have surface activity with the hydrocarbon chain dissolved in the apolar phase and with the polar head orienting toward the polar phase. Main reaction products, the FAME and glycerol distributes into apolar and polar phases.

Source E: Losses in refining crude biodiesel are similar to those described in feedstock refining. Trans-esterification reaction products separate into an upper apolar and a lower polar phase. Differential solubility components distribute between the phases. The presence of excess of reagent, intermediary products and minor components contribute to dragging further components into the byproduct stream. This solvent extraction effect is mainly attributed to the excess of reagent, presence of amphiphilic molecules and selectivity of the glycerol. It is necessary to improve selectivity of the refining agent. But the presence of intermediary components with surface active properties is unfavorable for efficient breakdown of the dispersed system. If the dispersed state is maintained, finely dispersed glycerol promotes hydrolysis and shifts the trans-esterification equilibrium away from complete conversion. More efficient separation of glycerol through colloid chemistry modifications would lead to greater biodiesel conversion.

Source F: For the sake of complete review it is to mention that significant losses can be generated through improper selection and use of additives in a colloid system.

A number of researchers have investigated the impact of col- 


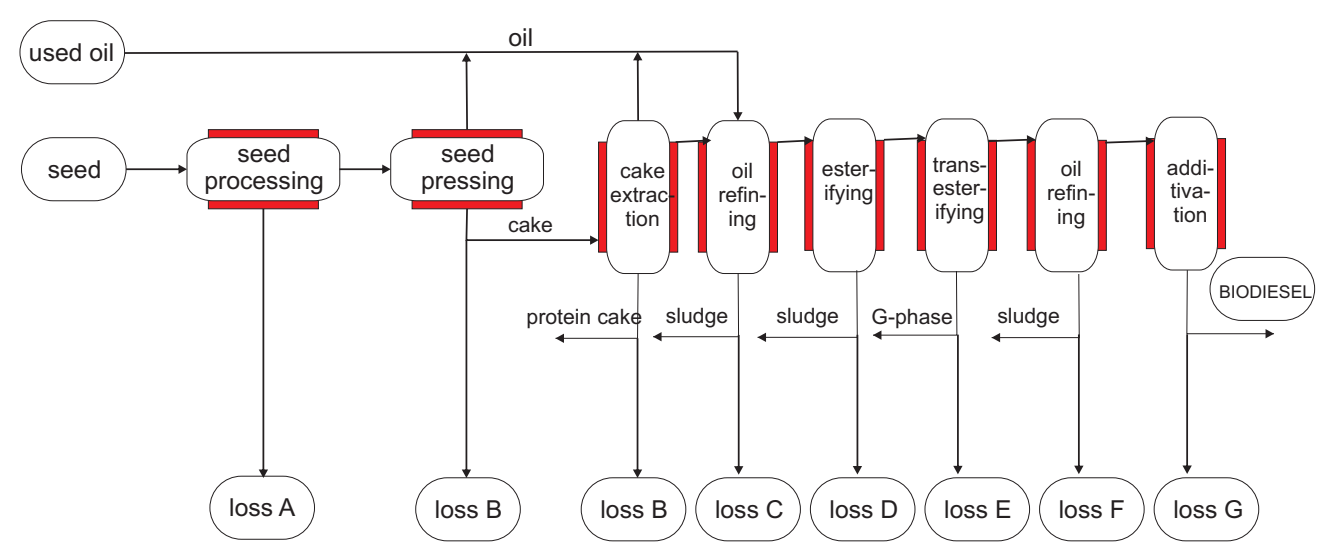

Fig. 1. Losses occurring in biodiesel production operations

Tab. 1.

\begin{tabular}{|c|c|c|c|c|}
\hline \multirow{6}{*}{$\begin{array}{l}\text { Trans- } \\
\text { esterification }\end{array}$} & $\mathrm{TG}+\mathrm{CH}_{3} \mathrm{OH}$ & methanolysis & - FAME +DG & \multirow{6}{*}{$r-1$} \\
\hline & & & hydrolyis & \\
\hline & & \multirow{4}{*}{$\begin{array}{l}\stackrel{\text { methanolysis }}{\longrightarrow} \\
\text { methanolysis }\end{array}$} & - FAMF +MG & \\
\hline & & & hydrolyis & \\
\hline & $\mathrm{MG}+\mathrm{CH}_{3} \mathrm{OH}$ & & $\longleftarrow$ FAME $+\mathrm{G}$ & \\
\hline & & & Myaronyis & \\
\hline \multirow[t]{3}{*}{ Saponification } & $\mathrm{R}-\mathrm{C}(\mathrm{O})-\mathrm{OH}+\mathrm{KOH}$ & $\stackrel{\text { saponification }}{\longrightarrow}$ & $\longleftarrow$ R-C $(\mathrm{O})-\mathrm{OK}+\mathrm{H}_{2} \mathrm{O}$ & \multirow{3}{*}{$r-2$} \\
\hline & & nonifinotion & hydrolyis & \\
\hline & $\mathrm{FFA}+\mathrm{KOH}$ & saponification & $\overleftarrow{\text { hydrolyis }} \mathrm{FAK}+\mathrm{H}_{2} \mathrm{O}$ & \\
\hline \multirow{3}{*}{ Esterification } & $\mathrm{R}-\mathrm{C}(\mathrm{O})-\mathrm{OH}+\mathrm{CH}_{2} \mathrm{OH}$ & methanolysis & (0) -0 & \multirow{3}{*}{$r-3$} \\
\hline & & & hydrolyis & \\
\hline & $\mathrm{FFA}+\mathrm{MeOH}$ & methanolysis & $\overleftarrow{\text { tydrovic }}$ FAMe $+\mathrm{H}_{2} \mathrm{O}$ & \\
\hline
\end{tabular}

loid chemistry on process operations, and have shown that colloid phenomena play an important role in the reaction kinetics of trans-esterification in conventional systems. For example, saponification is vital in conventional processes, since the soap formed is needed to kickstart the reaction [9], since the soap brings molecules of substrate oil, reagent and catalyst in contact, which would otherwise remain separated in distinct phases. Dorado et al [10] assessed the methods of optimizing FAME production and concluded that optimization was highly dependent on the FFA content of the feedstock, since rising amounts lower product yield and increase the specific consumption of energy and auxiliaries. Moreover any water present in the fuel increased the risk of formation of dispersions. Similarly Doell et al [11] have demonstrated the importance of the behavior of different phases in a series of trans-esterification reactions. Partially converted glycerides which form during trans-esterification having been concluded to be the surface active agents in the process of trans-esterificaation. Kickstarting role of these is not addressed, but the importance is accredited to maintaining the dispersed state throughout the whole process which controls the mass transfer in the biphasic system. We consider that both statements are correct, neither discredit the other. Both the composition and concentration of these surface active agents have a role in controlling interfacial processes. The surface active agent, whether an amphiphilic soap or glyceride, promotes the formation of finely dispersed system. In presence of the correct amount and activity of amphiphilic substances and under intensive mixing, the size of these dispersed globules can be reduced to the point where they are solubilized in the continuous phase [12]. However for efficient solubilization into microemulsions, the rate of methanol to oil must be multiple to practice of industrial biodiesel synthesis. In the presence of macroemulsions, intensive mixing during processing is necessary to bring the reaction components into contact. If this mixing is intensive enough and the composition and concentration of surface active agents are appropriate, methanol can be finely dispersed in oil to an extent that the system appears to be homogenous without true solubilization.

This means that the maintenance of permanent mixing during processing to promote breakage and reformation of the dispersed system is necessary. Stability of the emulsions is influenced by changes in the properties of the encapsulated matter in the chemical reaction. The droplets must break and the components must be able to transfer through the interface to be distributed according to their preferential solubility. Without a change in properties of the encapsulated matter, emulsions - whether macro- or micro- - can appear to be stable for the period of processing time. Residence times in a settler are typically longer than the stability of the dispersed system under the applied conditions. 
Use of a phase transfer catalyst (PTC) has a similar impact on mass transfer in trans-esterification as surface active agents in the dispersion do. Efficiency of PCT catalysts provides further details on the underlying mechanisms. Zhang et al [13] demonstrated that the addition of a PTC greatly increases transesterification reaction rates. From colloid chemistry perspectives, PTCs are and act as surface active agents, allowing mass transfer and promoting reaction through the interface. The catalyst having double function acts for phase transfer and chemical reaction catalysis alike. It is worth stressing that the time to equilibrium depends on the operational conditions and may not be true for different operational conditions. For example, conclusions drawn at exaggerated dilution levels may not be equally true for industrial conditions. Reaction partners come into intimate contact with the catalyst on the enlarged surface area of the interface, which is favorable for trans-esterification. The lower the droplet diameter the higher the surface area, meaning either the interfacial tension must be reduced or the intensity of mixing must be increased. Forward and backward reactions are equally possible if the mass transfer is maintained between the separated phases. Dispersion has similar effects on interfacial reactions.

Although these interpretations are supported by colloid chemistry, there are debates about the possible mechanisms and kinetics, with questions being asked about how the overall process is controlled. Mittelbach, Zhang and Berrios [14] argue for a homogeneous phase reaction on the grounds of the phase transition of the catalyst and reagent into the continuous phase. The interface properties control the mass transfer meaning the reaction is dependent on kinetic factors. However Negi et al [15] conclude that knowledge of the mass transfer and the reaction mechanism is still far from complete, including where the reaction exactly takes place, whether at the interface or in one or both the phases, in bulk or in film or in both of these forms. Most explanations link the underlying mechanism to the surface active property of molecules at the interface. This suggests to us to accept that the reaction is interfacial. Surface active components promote formation of a dispersed system. This is required to create a high surface area with reduced interfacial tension, and allow for mass transfer and intimate contact of reagent, catalyst and substrate. Soap and glyceride molecules are formed by the same mechanism. The substrate (FFA or TG) in the oil phase contacts the alkali in the methanol phase through the interface. These natural surfactant molecules in the feedstock are promoters of phase transfer and also encourage catalysis.

The rate of the trans-esterification reaction is frequently claimed to be slow and controlled by diffusion [16]. However this is only relevant for trans-esterfication conducted in biphasic systems. In homogenous systems, reaction rates do not depend on interfacial phenomena and rapidly reach equilibrium conditions $[8,9,17]$. The equilibrium of methanolysis - glycerolysis $\{r-1\}$ is controlled by the concentration profiles of the reaction partners at the reaction contact surfaces. Mixing is not selective, but may drive the reaction in either direction. Intensive (high shear) mixing is necessary to renew optimally the reactive contact of molecules in distinct phases, and shorten the time to equilibrium. Ultimately the process is controlled by the combined effects of mass transfer (mixing, interface renewal) and the chemical reaction. Control is exerted through modification of the interfacial surface tension conditions. Employing a high excess of reagent reduces the interfacial resistance, promoting both mass transfer and chemical reaction rates. Repeated phase transfer through the interface during trans-esterification explains the need for an optimum initial FFA content in the feedstock, with the optimum level depending on prevailing conditions. Intensive mixing makes the contact of reaction partners more likely, while excess of reagent moves the equilibrium toward the desired direction, but neither can boost the methanolysis to completion under standard affordable operational conditions.

The key to improving the biodiesel conversion process lies in modification of the interfacial interactions. Boocock [17] used colloid chemistry principles to eliminate interfacial resistance during trans-esterification and conducted a sequence of transesterification reactions in a homogenous phase. Others have recommended the use of different polar co-solvents, and there have been attempts to perform trans-esterification in supercritical conditions using this approach. In turn, we have performed experiments on the elimination of the interfacial resistance in trans-esterification and restituting the interfacial resistance for selective separation of main and byproducts into distinct phases.

Most conventional units achieve the necessary "coexistence" of reaction partners by increasing the degree of dispersion. Intensive high shear mixing creates contact events between the dispersed globules. As long as dispersed droplets form and reform in the presence of the reagents and come into intimate contact with the active homogeneous catalyst, the sequence of reactions can be sustained. As a consequence of the chemical reactions colloid chemical conditions change, the newly formed droplets can tear away valuable end products into the byproduct stream. These contribute to losses in product yield of a few percentage if reported to the amount of the feedstock and 10-20\% if reported to the amount of the byproduct phase [18]. Theoretically the entrapped oil can be released by phase inversion. With control of shear rate and concentration of surface active agents the diameter of the dispersed droplets can be reduced to a minimum where phase inversion occurs in macro-emulsions. But under the ratios employed in biodiesel synthesis this phase inversion is not possible.

Our aims in developing a process [19] on colloid chemistry grounds were:

- To promote the forward methanolysis reaction rate,

- To prevent the backward glycerolysis/hydrolysis reactions,

- To shift the equilibrium toward complete conversion in a single reaction step.

We found that these objectives can be met by the use of hexane solvent. There are a few references to the use of hexane, but 
these are not directly related to the synthesis and processing of biodiesel. These prior works have highlighted the value of using apolar solvents in refining the crude biodiesel, but failed to recognize any benefits during synthesis. In selected models [21], [22] hexane has been used to predict phase equilibrium and calculate the refining needs of idealized feedstock mixtures. The complex colloid nature of biodiesel feedstock and colloid chemistry phenomena in processing necessitates experimental proof of these theories. Our experiments provide data for demonstrating the potential for reducing losses and improving efficiency in biodiesel processing by the proper use of an apolar solvent to control phase transfer. In the present article we present data for phase behavior and the impact on trans-esterification, but these general conclusions can also be applied to the esterification of FFA.

\section{Experimental}

Two sets of experiments are presented in the present article: phase pattern and trans-esterification conversion experiments.

\section{Phase pattern experiments:}

Objective: To measure the amount of reagent readily available for trans-esterification $\left(\mathrm{R}_{a}\right)$. The amount of reagent readily available is expressed as the amount of methanol dissolved in the same phase as the substrate oil (F: feedstock).

Apparatus: Near equilibrium liquid-liquid extraction apparatus, consisting of a jacketed glass mixer settler, reflux condenser and doping funnel. Vessel sizes: 0.251 and 11 were used to meet analytical requirements and allow further processing. The stirring rate was kept moderate to avoid solubilization and emulsification. The temperature in the mixer settler was controlled by circulated hot water in the jacket. The reflux cooler condensed evaporated solvent and reagent to avoid loss of volatiles. Feedstock, solvent and reagents were introduced through a doping funnel. The interface between the phases was clearly visible for drainage.

Materials: Feedstock: Partially refined high oleic acid sunflower (HOSO) and rapeseed oil (RO) were used. Properties are listed in Table 1. The solvents and reagents were of technical grade. Solvents: polar solvent: N-methyl-2-pyrrolidon $\left(\mathrm{S}_{p}: \mathrm{NMP}\right)$ and apolar solvent: $\mathrm{n}$-hexane $\left(\mathrm{S}_{a}: \mathrm{nC}_{6}\right)$. Reagent: methanol ( $\left.\mathrm{R}: \mathrm{CH}_{3} \mathrm{OH}\right)$. The reagent acted as polar solvent component too. Distilled water in the range of $1-5 \%$ was added as a co-solvent to polar solvents to influence selectivity and solvency. The catalyst was not added to avoid methanolysis. Although spontaneous conversion was expected to be limited, methanolysis could not have been totally prevented. Rates of reagent to substrate (R:F) and solvent to substrate $(\mathrm{S}: \mathrm{F})$ were selected to match the typical ranges used in industrial processing.

Experimental procedure: A weighed amount of oil was mixed with the specified amount of solvent and poured into the mixer settler. The content was heated to experimental temperature using moderate stirring (300 1/min). On reaching the experimental
Tab. 2. Feedstock characteristics

\begin{tabular}{lcc}
\hline Feedstock designation & Sunflower "HOSO" & Rapeseed \\
\hline Loss on refining, \% & 8.8 & 4.3 \\
FFA content, \% & 1.8 & 1.3 \\
lodine number & 106 & 113 \\
Phosphorous, mg/kg & 82 & 43.4 \\
Viscosity @ 40 $40^{\circ} \mathrm{mm} / \mathrm{s}$ & 33.2 & 32.5 \\
Oleic acid, \% & 87.6 & 63.5 \\
\hline
\end{tabular}

temperature, the weighed stochiometric amount of reagent was added through the doping funnel. The mixture was stirred for 15 min. The relatively short stirring time was a compromise between perfect mixing for liquid-liquid equilibrium and avoidance of progress of the chemical reaction. After this the system was left still for 30 minutes at the same temperature. Upper apolar and lower polar phases were separated by draining. Reagent and solvent removal from the raffinate (upper) phase was done by extraction with water $(2 \times 10 \%)$ and distillation.

Analysis: Details are given in Table 3

\section{Trans-esterification:}

Objective: To study trans-esterification under controlled interfacial properties for the sake of increased reaction rates and shifted the equilibrium toward methanolysis.

Apparatus: A loop reactor was used for the experiments with an apolar solvent, while standard four neck glass flasks equipped with heating mantle, reflux cooler, overhead stirrer, metering funnel and sampling device were used in the experiments using no solvent or a polar solvent. The loop reactor system was specially designed for continuous separation of the glycerol formed during trans-esterification so full conversion in a single operational event could have been realized. Withdrawal of the glycerol phase was done after the conversion was completed. Heating and temperature control was applied through the jacket of the loop reactor. The loop reactor was packed with ceramic Rashig rings of $6 \mathrm{~mm} \times 6 \mathrm{~mm} \times 1 \mathrm{~mm}$. Mixing was controlled by the speed of the circulation pump. Control of reaction temperature was imposed by the boiling point of the reagent-solvent mixture $\left(\sim 50 / 67^{\circ} \mathrm{C}\right)$.

Materials: as in phase equilibrium tests and technical grade $\mathrm{KOH}$ for catalyst.

Experimental procedure: The substrate oil and solvent were poured into the reaction flask, or main vessel of the system and heated to reaction temperature. The calculated amount of reagent-catalyst mixture was added to the loop reactor system through the reflux cooler and through the doping funnel to the conventional system. The withdrawal height for circulation stream was at a point above the interface layer. Experiments were conducted for specified periods of times during which time the circulation/mixing was maintained. The transesterification reactions in the three neck flask apparatus were stopped by adding $10 \%$ water. In the loop reactor system, the 
circulation was stopped and the system was left to stand for 30 minutes. The lower glycerol phase was withdrawn, weighed and analyzed. The upper biodiesel phase was treated as in phase equilibrium tests and tested.

Analysis: Details are given in Table 3

\section{Results}

\section{Phase behaviour}

The amount of reagent readily available for the transesterification reaction $\left(\mathrm{R}_{a}\right)$ is presented in Fig. 2. expressed as the amount of methanol dissolved in the same phase as the oil substrate. Data points were calculated from mass balance data. Each curve represents data relevant for conditions close to biodiesel synthesis $\left(\mathrm{t}=50^{\circ} \mathrm{C}, \mathrm{p}=\right.$ atmospheric $)$. For complete conversion, the rate of $\mathrm{R}_{a}: \mathrm{F}$ (F:feedstock) must be at least 3:1 (stochiometry), i.e. all added reagent molecules are dissolved in the same phase as the feedstock.

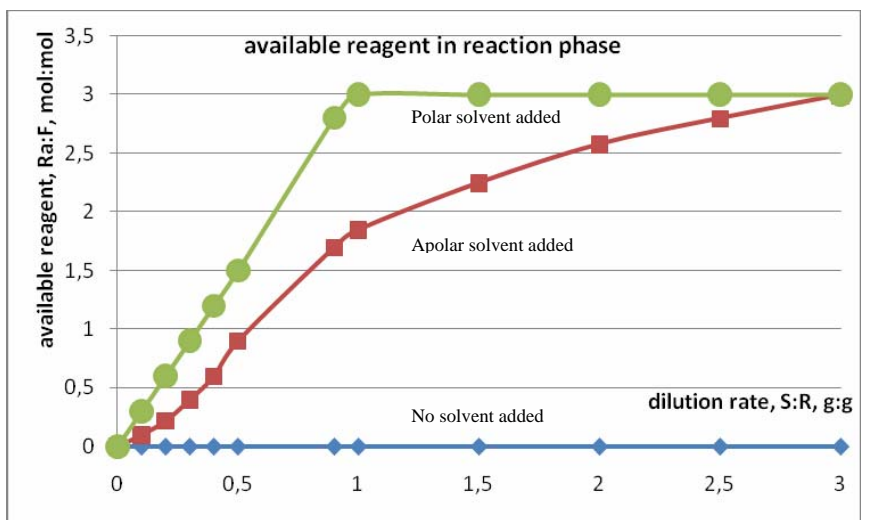

Fig. 2. Reagent readily available for trans-esterification

In absence of a solvent (no $S$ ), there is no readily available methanol in the same phase as the oil. Reagent methanol forms a distinct supernatant layer on top of the oil phase similar to excess methanol in a standard process. The probability of transesterification reactions under such conditions is insignificant. After mixing and settling, the light phase showed very slight change in colour. The amounts of components mutually dissolved in either phase were below the level of analysis, irrespective of the amount of excess methanol. This test confirmed that without a phase transfer agent, reaction partners are separated in distinct phases by a barrier interface. The readily available contact area is controlled by the geometry of the vessel. To achieve contact between the reagent and the substrate, intensive mixing and creation of a dispersing phase is essential.

Both polar $\left(S_{p}\right)$ and apolar $\left(S_{a}\right)$ solvents can remove the barrier by dissolving the reagent and substrate in a common phase. This allows reaction under conditions of biodiesel synthesis without the need for dispersed system created through vigorous mixing. Polar solvents, if heated close to reaction temperature, dissolve enough substrate to initiate trans-esterification in the reagent phase at a low rate. By adding a polar solvent to methanol in a ratio of $\mathrm{S}_{p}: \mathrm{R}=1: 1[\mathrm{~g}: \mathrm{g}]$, the system formed was mono-phasic at $55^{\circ} \mathrm{C}$. This level represents the lowest level of polar solvent $(10 \%)$ to the feedstock capable of producing a reaction in a single phase $S_{p}: F>1: 9$ [g:g]. Below this level, the system was biphasic and only part of the reagent was in the same phase as the feedstock. At lower temperatures and solvent rates, the polar solvent formed a bi-phasic system, with the solvent layer below the supernatant oil layer. The bottom layer showed signs of solvent extraction through the appearance of a slightly darker colour.

More apolar solvent additon was needed to achieve stochiometric availability of reagent to feedstock. The apolar solvent was able to dissolve both the reagent and substrate in a single liquid phase at a ratio of $\mathrm{S}_{a}: \mathrm{R}=3: 1$ [g:g] or above. This represents a dilution of the feedstock by $30 \%$ apolar solvent, $\mathrm{S}_{a}: \mathrm{F}>3: 7[\mathrm{~g}: \mathrm{g}]$. From this point on, the system became monophasic.

Above these limit concentrations $\left(S_{p}: F>1: 9\right.$ and $\left.S_{a}: F>3: 7\right)$ [g:g], the single phase condition could have been prevailed under the addition of an excess amount of reagent; hence transesterification can be influenced kinetically toward methanolysis.

Apolar and polar solvents have partial mutual solubility in each other. When added together, duo-sol type solvent refining of the substrate was performed. Fig. 3 shows the refining action of a duo-sol extraction of HOSO using a mixed solvent of $\mathrm{S}_{p}: \mathrm{F}=1: 9$ and $\mathrm{S}_{a}: \mathrm{F}=3: 7$ ) [g:g] in terms of the viscosity of the raffinate. The supernatant (raffinate) apolar layer consisted of hexane and the dissolved oil components and some methanol, while the lower (extract) layer of NMP dissolved the rest of the methanol and polar components. The split of phases was rapid on turning the mixer off, with a clear, sharp interface distinguishing the two phases. The upper phase became lighter, and the lower darker. The extract phase held the polar components by selective (preferential) solubility of polar and ionic components. The lighter, cleaner raffinate phase lost components known in vegetable oil processing practice as gums.

Similar to petroleum refinery practice, the addition of water as co-solvent increased selectivity and reduced solvency of the polar solvent. Because of the very limited solubility of water in hexane, the yield of the raffinate phase was due to the rejection of lower polarity oil components from the extract phase into the raffinate phase. This improvement in separation reduced loss, and accordingly led to an increase in oil yield. With HOSO, the loss was reduced from $8.8 \%$ to $2.9 \%$; with RO the loss was reduced from $4.3 \%$ to $2.7 \%$.

The effect of NMP containing 5\% water is presented in Fig. 3 in terms of change in viscosity of the oil. The ratio of feed:solvent $(\mathrm{F}: \mathrm{S})$ was calculated as $\left(\mathrm{F}+\mathrm{S}_{a}\right): \mathrm{S}_{p}$. In Fig. 3 the range of $F: S \approx 1: 1$ represents the case of stochiometric availability of the reagent as in Fig. 2 Similar refining activity was shown by tracking changes in specific gravity and phosphorous content. It should be noted that yields in duo-sol refining were better than figures reported for standard neutralization and acid degumming refining. 
In a series of experiments glycerol was added to the duo-sol system. This addition reduced selectivity and increased solvent power for polars in the polar phase of the duo-sol system. Glycerol acted as a secondary polar solvent $\left(S_{p},=S_{p}+G\right)$ and competed with hexane for dissolving methanol. A sharp drop in the amount of readily available reagent was observed even under high excess of reagent R:F =9:1 [mol:mol]. As opposed to what might be expected from solventless biodiesel synthesis, the increased use of solvent slowed the trans-esterification reaction in duo-sol solvent system.

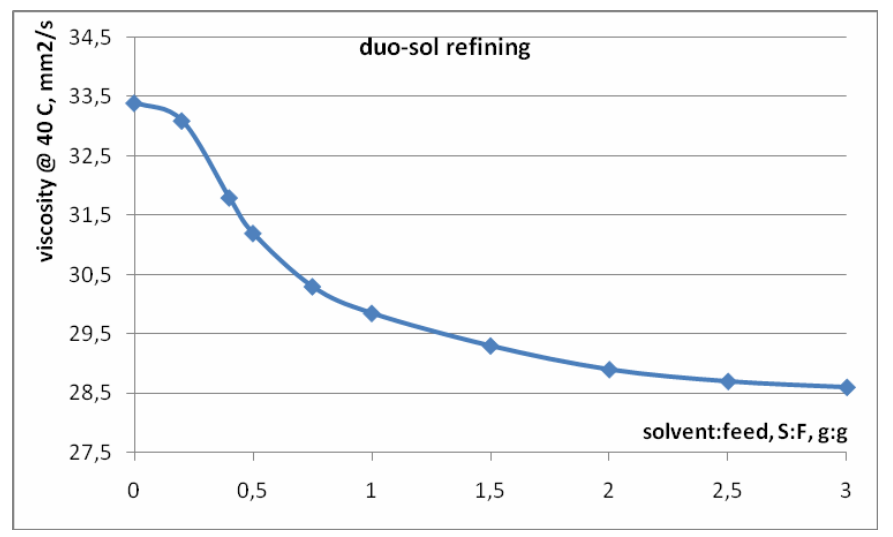

Fig. 3. Duo-sol refining of high oleic sunflower oil

\section{Trans-esterification}

In solventless trans-esterification, there is a need for intensive mixing. As known from practice, equilibrium conversion level can be reached in around 30 minutes in the absence of a solvent. An excess of methanol leads to methanolysis at a slightly higher equilibrium level, without significant improvement in the time to equilibrium. These equilibrium conversion kinetic curves are presented in Fig. 4 and constitute a basis for comparison for kinetic curves in the solvent assisted cases. Since the polar solvent systems keeps all components in a homogeneous mixture and the apolar solvent system rejects the polar constituents we figured both cases in a single chart.

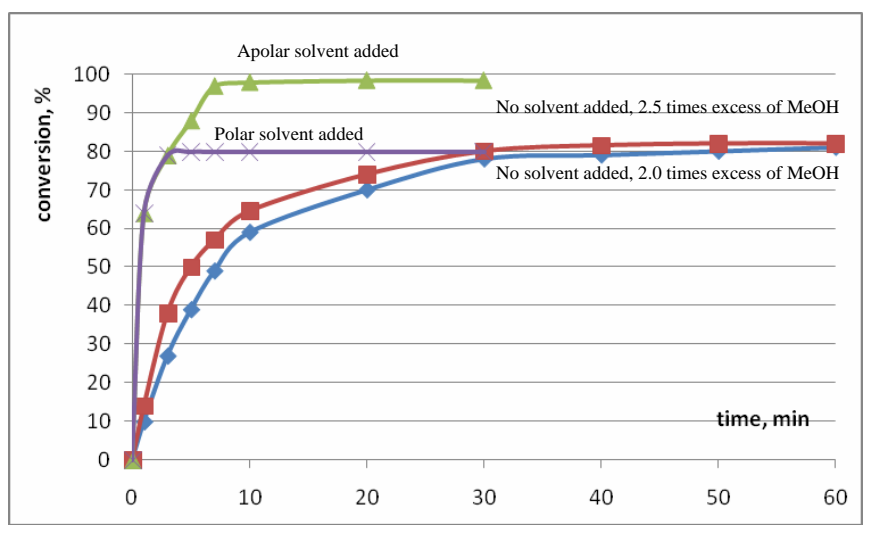

Fig. 4. Trans-esterification kinetics

The polarity of a solvent has no difference on the ability to boost reaction kinetics. Both types of solvent provoked signif- icantly faster conversion to equilibrium conditions within 3-10 minutes. The kinetic curves overlapped for the first 3 minutes, since the readily available reagent within the reaction media is in excess, while in solventless cases the availability is scarce. To maintain the reaction the reactive interface must be renewed, so a high degree of dispersion promotes the interfacial mechanism for both the forward and backward reactions. The time to equilibrium depends on the ease of renewal of the reactive interface. This is achieved by penetration of the apolar hydrocarbon chain of the amphiphilic molecule into the bulk of the continuous oil phase. Thanks to this positioning between the two phases, the charged polar function group can act to keep the reagent/reactant molecules close enough to the continuous phase to allow intimate contact of reaction partners. The reduced interfacial tension promotes faster formation and reformation of the dispersed globules making it necessary to apply shear conditions (mixing) to renew contact between the reaction partners separated in distinct insoluble liquid phases.

For the sake of separation of target fuel components and byproducts both must leave the active site. This requires mass transfer through the interface. Without this interfacial mass transfer the chemical equilibrium in $\{r-1 . .3\}$ would establish within the entrapped globule, preventing full conversion. For formation and reformation of dispersed globules it is beneficial to reduce the interfacial resistance. It can be done by increase of temperature and pressure, rates of reactant, use of co-solvents. Surface active agents are amphiphilic and by such they can be beneficial or detrimental, depending on conditions and rate of application. Majority of natural surface active agents present in feedstock (phospholipids) and those formed during the sequences of reactions $\{r-1 . .3\}$ (soaps, mono- and glycerides) reduce the interfacial tension. Other components called gum constituents exert surface activity but because of having role to stabilize the droplets prevent mass transfer and obstructs the desired conversion. Inadequate feedstock pretreatment can stabilize losses by tight entrapment of the target products in droplets. These droplets merge to form the byproduct layer without the breakage of the internal structure that keeps the target product solubilized within the phase. This solubilization is similar to apparent solution of methanol in oil after intensive high shear mixing. The resulting phase resembles the appearance of solution, but is a fine dispersion. The progress in conversion changes permanently composition of phases and properties of the interface. Overall reaction kinetics and conversion can be influenced if these colloid phenomena are controlled.

The fate of intermediary products and byproducts (glycerides and glycerol): reaction products of (trans-)esterification separate into an upper apolar and a lower polar phase, just like in solvent extraction. Differential solubility components presents in the overall system results in a distribution of these between the phases. The presence of excess of reagent, intermediary products and minor components of the feedstock make that some dissolve in both phases and drag further components to 
be distributed between the phases. This solvent extraction effect is mainly attributed to the excess of reagent, presence of amphiphilic molecules and selectivity of the glycerol. Any engineering solution must take into account solvent extraction in the byproduct stream when handling with biphasic systems.

Intermediary products (mono- and diglycerides) and side reactions of minor component (soaps and phosphorous compounds) are compounds with amphiphilic properties. Overall concentration profile of these change along the progress of chemical reactions. Because of amphiphilic character these molecules form a bridge between the different polarity phases. In the case of mono and diglycerides the fatty acid chain is dissolved by the oil phase, whilst the polar glycerine backbones sit in the dispersed phase. When the two phases are separated into a fuel and a glycerol phase, these amphiphilic molecules can drag oil components into the byproduct phase. The presence of intermediary components with surface active properties is beneficial for initiating the chemical reaction of molecules sitting in different phases and unfavorable for efficient breakdown of the dispersed system. If the dispersed state is maintained, finely dispersed glycerol promotes hydrolysis and shifts the trans-esterification equilibrium away from complete conversion. More efficient separation of glycerol through colloid chemistry modifications would lead to greater biodiesel conversion.

However the polarity of the solvent has a marked influence on the point of equilibrium. Polar solvent containing reaction systems reached equilibrium level similar to cases without a solvent. This is because the ratios of reagent to substrate and reaction conditions were similar and equilibrium conditions stabilized accordingly in response to reaction conditions. In presence of the polar solvent the readily available reagent for the reverse reactions is dissolved in the homogeneous reaction phase. In solventless case the intensive mixing maintains equal probability for forward and backward reactions in the finely dispersed system. Availability of byproducts to drive the reverse hydrolysis reaction is assured in both cases. This availability is due to vigorous mixing in conventional biphasic systems and through maintaining a single phase by preferential solubility in polar solvent systems. The apolar continuous phase rejects the formed byproduct glycerol into a distinct polar phase. Due to the solvent, the byproduct is efficiently excluded from reaction zone and the reverse hydrolysis reaction is highly suppressed. To confirm this, the reaction was repeated in a continuously stirred four neck flask. The level of conversion equilibrium was similar to the levels seen in the two other cases. To shift the reaction equilibrium in the desired direction, an apolar solvent and an appropriate reaction technique are required. Settling rate of glycerol phase resembles the shape of emulsion breaking kinetics (Fig. 5). This slow rate makes impossible to shift the equilibrium toward methanolysis in conventional systems, even if the high shear mixing would have been lifted periodically. This is why the CD Process of Connemann [23] employs centrifuges for more intensive settling. Addition of an apolar solvent increases drastically the settling rate of glycerol, according to Stoke's law. The viscous retention of dispersed particles depends on viscosity of the medium, difference in specific gravity of the phases and radius (r) of the spherical body for gravitational forces to overcome diffusion. Addition of hexane to the oil results in reduced viscosity and specific gravity of the continuous phase, making viscous forces weaker. In addition these changes make glycerol droplets coalesce into larger droplets. As a result, the settling rate of glycerol droplets becomes fast enough in the loop reactor to prevent reverse hydrolysis efficiently. Addition of more apolar solvent promotes even faster rejection of glycerol. This helps shift the reversible trans-esterification toward complete conversion, making it possible to produce biodiesel in a single transesterification step. Similar shift cannot be achieved with a polar solvent, because the glycerol stays dissolved in the reaction phase. To separate glycerol for complete conversion, the solvent must be removed first. This involves an additional operation between the steps of trans-esterification - preferentially using distillation to separate the solvent (see Fig. 8a).

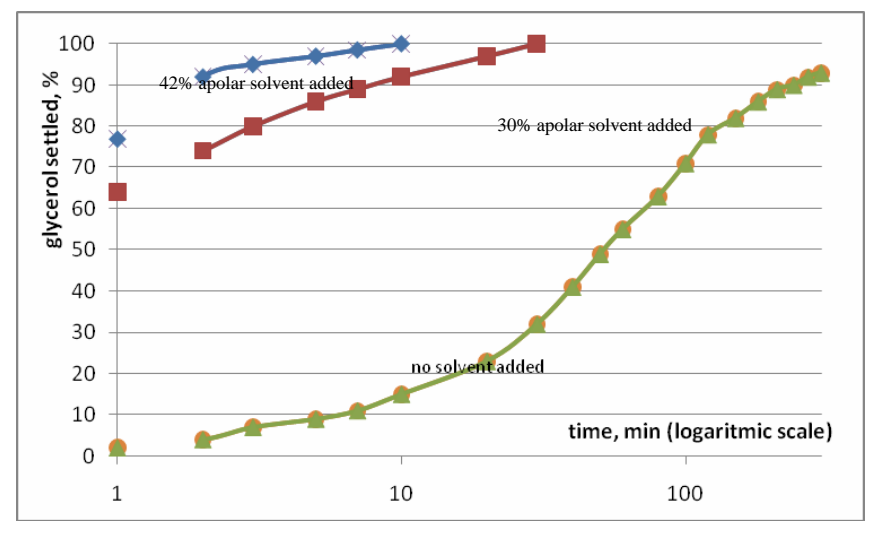

Fig. 5. Settling rate of glycerol at equilibrium conversion

There was no need for intensive mechanical mixing in the loop reactor system. Boiling and circulation of the fuel phase through the loop reactor conferred adequate mixing and progression to achieve full conversion.

According to the phase behavior tests, both polar and apolar solvents were capable of removing the interfacial resistance and bringing the trans-esterification reaction partners into intimate contact without the need to form a dispersed system by extensive mixing. The presence of readily available reagent in the substrate phase positively influences the rate of trans-esterification.

\section{The influence of water (Figs. 6 6and 7 )}

The system responses to the presence of water in transesterification depended on the presence and type of solvent. Trans-esterification was frozen in the system without a solvent.

Trans-esterification reactions could be sustained in the presence of water in solvent assisted systems. Up to $0.8 \%$, the cosolvent action of water was beneficial in increasing the FAME yield in the polar solvent system. Above this level, the presence 


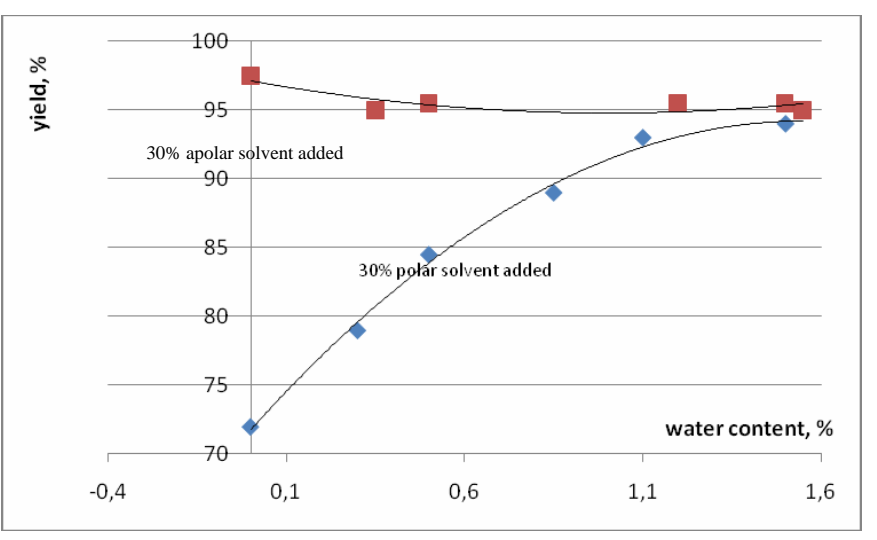

Fig. 6. Effect of water on biodiesel yield in trans-esterifying rapeseed oil.

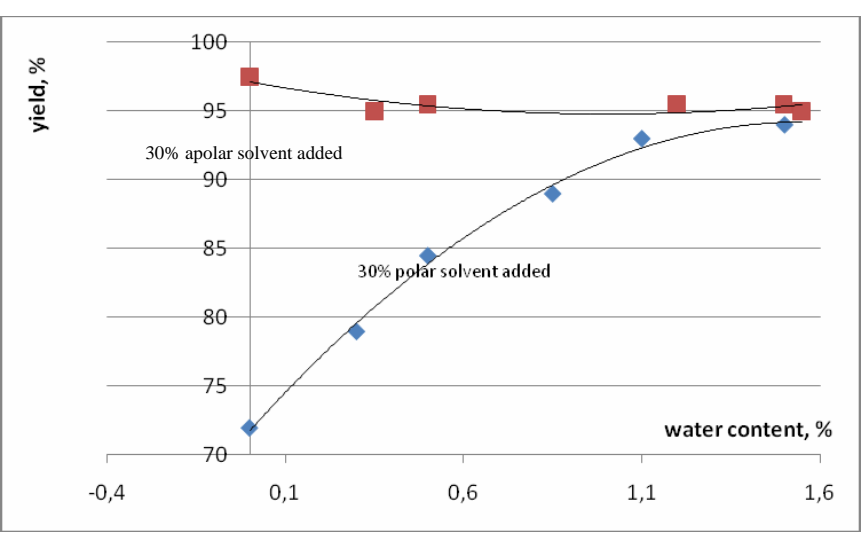

Fig. 7. Aggregate response of conversion*yield to water content

of water was detrimental under the applied experimental conditions.

Water acted as a co-solvent in apolar solvent system too. It competed for the reagent and catalyst in the dispersing phase and obstructed the conversion and hence decreased product yield.

The impact of water was less negative in apolar than in polar solvent. The higher yield of biodiesel product in the apolar solvent system was likely due to the difference in solubility. This is due to more efficient rejection of water from the reaction phase, as opposed to the water being dissolved in the homogeneous system.

The loss in yield in the apolar solvent system was associated with a prolongation of the components in the dispersing phase. The rejected glycerol and water formed lower specific density globules, which had slower settling rates, meaning the potential to prevent hydrolysis becomes weaker. The decrease in biodiesel product yield was directly related to the increase in water content.

The extent of the yield reduction was lower for fully refined degummed rapeseed oil. In trans-esterification of fully refined vegetable oils, yield and conversion curves were not dependent on water content in a system with apolar solvent within the range of interest. It is possible that the presence of phospholipids and soaps promoted formation of dispersions that slowed the rate of separation of glycerol and allowing hydrolysis in $\{r-1\}$. The almost complete rejection of water into the glycerol phase main- tained a "dry" reaction phase, favorable only to methanolysis.

In the polar solvent system, the yield of biodiesel was improved at the expense of a drastic reduction in conversion. Water exerted this influence through modifying the selectivity of the solvents dissolving selectively the byproduct, just like in practice of solvent extraction. Product and feedstock molecules were rejected into a distinct apolar phase and by such separated from the reagent and catalyst by an interface. To establish similar phase ratios in polar and apolar systems, the amounts of reagent and solvent to feedstock were significantly lower than previously reported for polar solvent systems. By adding polar solvent in sufficiently high amounts, this split into polar and apolar phases can be avoided. An aggregate figure of yield*conversion (Fig. 7) pinpoints the importance of water content on potential loss in operation.

A comparison of mass balances and product qualities is given in Table 4. Biodiesel synthesized with assistance of either of the solvents met biodiesel quality requirements set by ISO EN 14214 specifications. There are three marked differences: yield, phosphorous and water content. All are related to colloid chemistry features. The yield improvement of $1-2 \%$ can even provide the necessary margin for units that had to stay idle because of inadequate profit generation. The lower phosphorous and water contents cannot be directly translated into feasibility figures, but provide a reasonable reserve in blending of automotive diesel fuel.

\section{Discussion}

Both polar and apolar solvents offer advantages in improving efficiency and productivity in biodiesel synthesis. Colloid chemistry and selective solubility can explain the basic mechanisms which underlie the differences in outcomes between the two types of solvent.

Apolar solvents' advantages are the improvement in yield and the simplification of production. The selected solvent is similar to the solvent used to extract residual oil from pressed seeds. The use of the same apolar solvent makes possible to share the duty of solvent recycle between the oilseed processing and biodiesel processing units. This has a definite influence to reducing the capacity to scale. Smaller capacity units can afford to invest in solvent extraction and to increase the access to lower cost feedstocks.

In systems with an apolar solvent, a selective polar solvent (glycerol) is generated through a sequence of chemical reactions. The overall mechanism turns into a chemical reaction with an associated solvent extraction. The ultimate improvement in biodiesel yield is a result of the selectivity of solvents in dissolving the reaction partners and products and rejecting secondary products. Secondary products and polar components that are detrimental to biodiesel are dissolved in the rejected phase.

Exploring the solubility of solvents makes it possible to improve the efficiency of chemical reactions in biodiesel synthesis using basic colloid chemistry. The use of suitable solvents can 


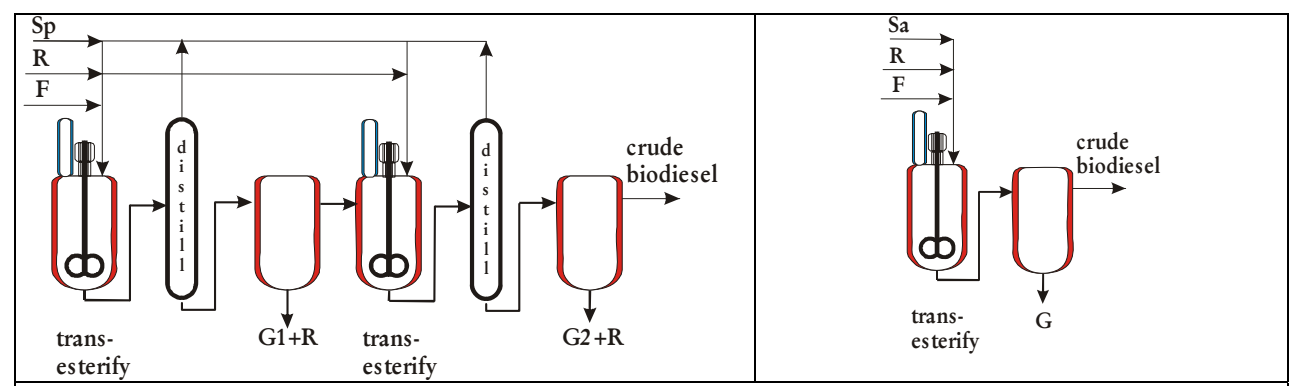

Fig. 8. Sequences of trans-esterification

Tab. 3. Analytical methods employed

\begin{tabular}{lll}
\hline Analysis & ISO standard & specifics \\
\hline Methanol content & EN 14110 & headspace chromatography. ACME 6100 Chromatograph, FID detector,
\end{tabular}
ACME 600 automatic headspace autosampler in split mode. HP1-HS capillary column of $0.32 \mathrm{~mm} I D, 30 \mathrm{~m}$ length, $0.1 \mathrm{~mm}$ film thickness. Reagent grade propan-2-ol added as internal standard. Temperature of injection and detection: $150{ }^{\circ} \mathrm{C}$, helium carrier gas at $40 \mathrm{kPa}$. The mixture of sample and standard was heated to $80^{\circ} \mathrm{C}$, maintained at this temperature for $30 \mathrm{~min}$. with frequent, automatic mixing.

\begin{tabular}{lll}
\hline Viscosity & EN 3105 & At $40{ }^{\circ} \mathrm{C}$ \\
\hline Acidity & EN 14104 & KEM AT 510 Automatic Potentiometric titrator \\
\hline Water content & EN 12937 & KEM 501 Automatic Coulometric Karl Fischer titrator \\
\hline $\begin{array}{l}\text { Specific gravity } \\
\text { Loss in refining }\end{array}$ & 3675 & \\
$\begin{array}{l}\text { Phosphorous } \\
\text { Glycerides }\end{array}$ & EN 14107 & Alfa Laval methods No 121 \\
& EN 14105 & on-column injection, oven profile as recommended by the standards.
\end{tabular}

Tab. 4. Biodiesel product and production characteristics

\begin{tabular}{llllc}
\hline Feedstock designation & \multicolumn{2}{l}{ Sunflower "HOSO" } & Rapeseed & \\
\hline Solvent used & Polar & Apolar & Polar & Apolar \\
\hline Number of trans-esterification steps & 2 & 1 & 2 & 1 \\
Biodiesel yield, \% & 96.6 & 98.4 & 97.1 & 98.8 \\
Viscosity @ 40 $\mathrm{C}, \mathrm{mm}^{2} / \mathrm{s}$ & 4.42 & 4.34 & 4.56 & 4.54 \\
Glyceride content, \% & $<0.01$ & $<0.01$ & $<0.01$ & $<0.01$ \\
Mono-Glyceride content, \% & 0.11 & 0.06 & 0.12 & 0.08 \\
Di-Glyceride content, \% & 0.06 & 0.02 & 0.04 & 0.02 \\
Tri-Glyceride content, \% & 0.07 & $<0.01$ & 0.08 & $<0.01$ \\
lodine number & 107 & 106 & 115 & 114 \\
Phosphorous, mg/kg & 8 & 5 & 10 & 4 \\
Acidity, mg KOH/g & $<0.5$ & $<0.5$ & $<0.5$ & $<0.5$ \\
Water content, mg/kg & 170 & 32 & 142 & 48 \\
Methanol, \% & $\mathrm{Nil}$ & $\mathrm{Nil}$ & $\mathrm{Nil}$ & $\mathrm{Nil}$ \\
\hline
\end{tabular}

modify the interfacial barrier between reaction partners. This is a necessary condition for achieving intimate contact between reaction partners. It can be achieved without intensive mixing and without the use of an efficient phase transfer agent.

Faster reaction kinetic rates constitute an argument in favor of diffusion control in methanolysis. The Stokes-Einstein relation explains this. Diffusion of components in a simple liquid phase is proportionally increased by lowering viscosity of the liquid. Einstein's viscosity equation explains that dilution has a minor influence in comparison to the extent of reduction in the hydrodynamic volume of diffusing elements. Dilution brings constituents in solution rather than in the dispersed system. The molecular radius of the largest tri-glyceride is in the order of angstroms, while that of a dispersion is in the order of microns (1 $\mu \mathrm{m}=10000 \AA)$. Under such influence the diffusion of the reaction components to and reaction products from the active site 
of the catalyst is intensified. Addition of excess reagent to promote diffusion in a homogeneous, solvent assisted system has less influence than in solventless systems.

In solvent-based systems, chemical reaction kinetics control the overall progress of biodiesel synthesis through the reduction in diffusion resistance. Equilibrium is reached very quickly when the solvent is polar, but because of the limited conversion, further synthesis with upstream separation of the solvent and byproduct is required.

Chemical equilibrium is reached slightly slower in apolar solvent, but conversion is close to completion in a single contact operation, with the process being controlled by both reaction kinetics and diffusion. The final control is exerted by formation of the rejection phase, with the byproduct diffusing not only from the active site of the catalyst into the bulk of a homogeneous system, but also crossing the newly formed interface.

The ratio of solvent to feed $(\mathrm{S}: \mathrm{F})$ is an important factor in evaluating reaction rates. Significantly lower amounts of apolar solvent are needed $\left(\mathrm{S}_{a}: \mathrm{F} \ll \mathrm{S}_{p}: \mathrm{F}\right)$. The controlling step in trans-esterification in an apolar solvent is the rate of separation of glycerol. Further improvement is very unlikely through increasing the ratio of $\mathrm{S}_{a}: \mathrm{F}$ within normal ranges. Ultimately the conversion rate from feedstock to refined biodiesel is the fastest in an apolar system.

The difference in conversion progress suggests that the action mechanisms for polar and apolar solvents are dissimilar. Polar solvents remove interfacial barrier between reaction partners and reaction products alike. Reaction partners and reaction products maintain diffusion to and from the active site in a homogeneous phase, with conditions favoring reversibility of $\{\mathrm{r}-$ 1 . There are no means of extending the level of conversion to close to completeness under normal operational conditions.

Apolar solvents remove the interfacial barrier between reaction partners in similar manner, but insert another interface separating reaction partners and products from the byproduct glycerol. Under normal operational conditions, conversion can be brought close to completeness. In an apolar solvent system the overall trans-esterification can be made unidirectional:

$$
\mathrm{TG}+3 \mathrm{CH}_{3} \mathrm{OH} \Rightarrow \quad 3 \mathrm{FAME}+\mathrm{G}
$$

The ejected glycerol has a selective solvent function, extracting polar constituents from the continuous phase of the reaction, which are detrimental to product quality. Similar extraction takes place after removal of the polar solvent splitting the homogeneous reaction product phase.

The overall reaction mechanism in apolar solvent systems is similar to solventless execution the synthesis: a solvent extraction associated chemical reaction. This makes the G-phase dark in colour. The combined solvent action of glycerol and methanol leads to lower selectivity and higher loss of main product in the byproduct phase in conventional systems, which results in loss of biodiesel in the G-phase. In samples from operational units not using a solvent, it is common to find partially converted feedstock and 10-20\% biodiesel in the glycerol phase. This represents a $1-2 \%$ loss of feedstock. By using an apolar solvent, this figure is reduced to below $0.1 \%$. Similar losses are inevitably found in the refining of crude biodiesel, particularly because of intensive mixing and presence of surface active agents (soaps, mono- and di-glycerides).

The optimum level of solvent usage dictates different strategies for using polar and apolar solvent. The preferential solubility of polar solvents for dissolving polar and ionic components and rejecting apolars must be corrected by a high ratio of $\mathrm{S}_{p}: \mathrm{F}$ to avoid formation of a biphasic system and loss of any valuable component. The preferential solubility of apolar solvents for dissolving reaction partners and biodiesel products and rejecting polars means the ratio of $\mathrm{S}_{a}: \mathrm{F}$ is irrelevant, meaning that $\mathrm{S}_{a}: \mathrm{F} \ll \mathrm{S}_{p}: \mathrm{F}$ is possible.

The presence of water has different effects in apolar and polar solvent systems. Water is formed in reaction $\{r-2\}$ irrespective of the solvent type. Addition of a small amount of water [0.1$0.8 \%$ ] raises the yield and conversion in polar system. Given the amount of water formed is about $1 / 20$ of the FFA content, polar solvent assisted systems are less prone to FFA content than conventional systems. Even a $10 \%$ FFA content feedstock produces water which falls within the range of having a beneficial effect on yield and conversion. Any glycerol byproduct is maintained in the mono-phasic system under such conditions. Addition of more water increases the selectivity of the polar solvent, leading to increased rejection of the substrate and separation of the reaction partners through the reformation of a barrier interface. This feature is exploited in solvent extraction techniques used in petroleum refining.

The beneficial features of using an apolar solvent are illustrated in Figs 8 . The series of operations in polar solvent system (figure 8.a) necessitates removal of the solvent to achieve separation of the byproduct. Within normal limits of current operations this is done by high specific energy consumption distillation of the light boiling-point solvent. The sequence of operations includes distillation in addition to the known structures used for solventless trans-esterification.

The system is simpler if an apolar solvent is used (Fig. 8p). We have sampled G-phases from several conventional biodiesel operations and found that the loss of yield is $1-2 \%$ of the feedstock. G-phases contained 10-20\% oil. In comparison, oil content of G-phase in apolar solvent system is below $1 \%$. Higher selectivity of the solvent for apolar compounds is beneficial in downstream crude biodiesel refining on the same basis. Losses are controlled by rejection of glycerol which results in a cleaner fuel stream and greater recovery of valuable components.

\section{Conclusions}

1 The use of apolar solvent improves biodiesel synthesis through modifications in colloid chemistry. 
2 A reduction in complexity (i.e. the number of unit operations) and processing time makes it possible to reduce the footprint of the biodiesel synthesis process area. Symbiosis of oil extraction and biodiesel processing become possible through use of similar solvent.

3 Overall process efficiency is increased by reducing production losses through by boosting reaction kinetics and mass transfer and improved separation of the fuel and glycerol phases. This results in better product yields and better quality products.

\section{References}

1 Razon L F, Alternative crops for biodiesel feedstock, CAB Reviews: Perspectives in agriculture, veterinary science, nutrition and natural resources 4 (2009), 56, DOI 10.1079/PAVSNNR20094056.

2 Bacovsky D, Körbitz W, Mittelbach M, Wörgetter M, Biodiesel Production: Technologies and European Providers, 2007. IEA Task 39 Report T39B6.

3 Tesser R, Casale L, Verde D, Serio D., Santacesaria E, Kinetics of free fatty acids esterification: batch and loop reactor modeling, Chemical Engineering Journal 154 (2009), 2533, DOI 10.1016/j.cej.2009.03.010.

4 Craig S, Production of hydrocarbons with a relatively high cetane rating, 1991. US Patent 4992605.

5 Boshle B M, Subramanian R, New approaches in deacidification of edible oils - a review, Journal of Food Engineering 69 (2005), 481-494, DOI 10.1016/j.jfoodeng.2004.09.003.

6 Aryusuk K, Puengtham J, Lilitchan S, Jeyashoke N, Krisnangkura K, Effects of Crude Rice Bran Oil Components on Alkali-Refining Loss, Journal of the American Oil Chemists' Society 85 (2008), no. 5, 475-479, DOI 10.1007/s11746-008-1215-0.

7 Dorsa, Eiickhoff, Basics of alkali refining of vegetable oils, available at http://stage.iupac.org/originalWeb/symposia/proceedings/ Tunis04/eickhoff.pdf

8 Erickson D R (ed.), World Conference on Edible Fats and Oils Processing: Basic Principles and Modern Practices, AOCS Press, 1990.

9 Mittelbach T, Kinetics of alkaline catalyzed methanolysis of sunflower oil, Fat Science and Technology 92 (1990), no. 4, 145-148, DOI 10.1002/lipi.19900920405.

10 Dorado M P, Ballesteros E, López F J, Mittelbach M, Optimization of Alkali-Catalyzed Transesterification of Brassica Carinata Oil for Biodiesel Production, Energy Fuels 18 (2004), no. 1, 77-83, DOI 10.1021/ef0340110.

11 Doell R, Konar S K, Boocock D G B, Kinetic parameters of a homogeneous transmethylation of soybean oil, JAOCS 85 (2008), 271-276, DOI 10.1007/s11746-007-1168-8.

12 Shinoda F, Emulsions and solubilizations, John Wiley and Sons, 1986.

13 Zhang Y, Stanciulescu M, Ikura M, Rapid transesterification of soybean oil with phase transfer catalysts, Applied Catalysis A 366 (2009), 176-183, DOI 10.1016/j.apcata.2009.07.001.

14 Berrios M, Martín M A, Chica A F, Study of esterification and transesterification in biodiesel production from used frying oils in a closed system, Chemical Engineering Journal 160 (2010), 473-479, DOI 10.1016/j.cej.2010.03.050.

15 Negi D S, Sobotka F, Kimmel T, Wozny G, SchomäckerR, Liquidliquid phase equilibrium in glycerol-methanol-methyl oleate and glycerolmonoolein.ethyl oleate ternary systems, Ind. Eng. Chem. Res. 45 ( 2006), 3693-3696, DOI 10.1021/ie051271r.

16 Noureddini H, Zhu D, Kinetics of transesterification of soybean oil, Journal of the American Oil Chemists Society 74 (1997), no. 11, 1457-1463, DOI 10.1007/s11746-997-0254-2.

17 Boocock D G, Konar S K, Mao V, Lee C, Buligan S, Fast Formation of High-Purity Methyl Esters from Vegetable Oils, JAOCS 75 (1998), 11671172, DOI 10.1007/s11746-998-0130-8.

18 Kocsisova T, Cvengros, G-phase from methyl ester production-splitting and refining, Petroleum and Coal 48 (2006), no. 2, 1-5.

19 Kovács, Improvements in or relating to a method for transesterifying vegetable oils, PCT/HU02/00114, WO/2003/040081, US 20050016059, CA 2466489, EP 2002777586, 2002.

20 Kovács, Transesterification of vegetable oils PCT/GB2009/000246, WO/2009/095668, 2008.

21 Oliveira M B, Queimada A J, Coutinho J A P, Modelling of biodiesel multicomponent systems with the cubic-plus-association (CPA) equation of state, Ind. Eng. Chem. Res 49 (2010), 1419-1427, DOI 10.1021/ie901264d.

22 Tizvar R, McLean D D, Kates M, Dubé M A, Liquid-liquid equilibria of the methyl oleate-glycerol-hexane-methanol system, Ind. Eng. Chem. Res 47 (2008), 443-449, DOI 10.1021/ie070882b.

23 Connemann, Process for the continuous preparation of c1 to c4 alkyle esters of higher fatty acids. Leer Connemann Oelmuehle September 1993 EP0562504. 